\title{
Registre du cancer et soins en oncologie
}

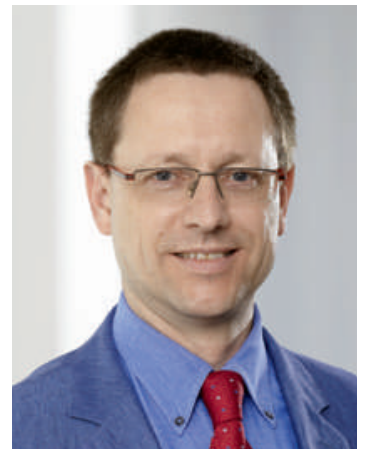

Mettre en réseau des informations au profit de la qualité des soins revêt une importance majeure pour la FMH. C'est ainsi qu'en fondant l'Académie suisse pour la qualité en médecine (ASQM) le 27 novembre 2012, la FMH a posé un jalon important en vue de consolider son rôle de «connecteur» aussi bien entre les médecins qu'au-delà du corps médical. Pour elle, la primauté des sociétés médicales dans toutes les questions spécifiques à leur discipline est incontestée, c'est même une condition sine qua non, au vu de l'importance du rôle qu'elles assument. Même si de temps à autre des divergences de vue apparaissent, qui peut, mieux que les sociétés de discipline, connaître les enjeux liés à leur spécialité et qui est le plus à même de les pallier?

\section{Les brefs délais accordés lors de l'audition prêtent à croire que l'avis des experts consultés n'intéresse pas vraiment.}

Le 7 décembre 2012, la Confédération a mis en consultation l'avant-projet de loi fédérale sur l'enregistrement des maladies oncologiques, en fixant le délai de réponse au 22 mars 2013. Nous estimons très positive la proposition de non seulement réglementer la saisie des données mais également de l'harmoniser et de la transcrire dans un registre national. La Société suisse d'oncologie médicale (SSOM) propose de s'investir dans ce projet en compilant les données du registre fédéral dans un registre propre à la société de discipline. Cela lui permettrait de les coupler avec les données des prestataires de soins et de les mettre à la disposition de ses membres afin de garantir la qualité élevée des soins. Cependant, il serait souhaitable de réaliser une étude de faisabilité.

Dans le cadre de la mise en œuvre de la Convention intercantonale relative à la médecine hautement spécialisée (CIMHS), l'instance en charge de la médecine hautement spécialisée (MHS) auprès de la Conférence suisse des directrices et directeurs cantonaux de la santé (CDS) a ouvert, le 18 décembre 2012, une procédure d'audition relative aux traitements des maladies oncologiques rares de l'adulte. Cette audition se terminera le 22 janvier 2013. Ratifiée par les 26 cantons, la CIMHS a indéniablement un caractère législatif. Le projet de planification est assimilable à une ordonnance, le délai de la consultation devrait donc être de trois mois. En raison du délai très court qui - nota bene - inclut les fêtes de fin d'année, on est en droit de se demander si l'intérêt accordé par la CIMHS aux avis des experts est réellement sérieux. Vous trouverez des informations relatives à la prise de position de la SSOM à la page 94 de ce numéro du Bulletin des médecins suisses.

\section{Une plateforme nationale serait plus judicieuse que différents centres de compétences en oncologie.}

En outre, la FMH porte un regard sceptique sur la planification de sept centres de compétences, qui risque de cimenter un éclatement structurel de notre pays relativement petit. Il serait beaucoup plus judicieux de créer une plateforme nationale, avec un soutien international et un concept de processus thérapeutiques basé sur des guidelines nationaux et internationaux. Par ailleurs, la volonté desdits centres d'élargir leur champ d'action au domaine ambulatoire va à l'encontre du mandat légal stipulé à l'art. 39 LAMal; elle restreint également la liberté thérapeutique des soins ambulatoires qui, aujourd'hui, représentent $90 \%$ des traitements, et réduit le libre choix du médecin. Cela est d'autant plus stupéfiant que les citoyens se sont nettement exprimés en 2008 et en 2012.

La FMH s'engage pour une formation médicale efficace et de qualité élevée. C'est la raison pour laquelle l'Institut suisse pour la formation médicale postgraduée et continue (ISFM) ainsi que l'ASMAC et l'AMDHS doivent également être entendus.

Enfin, pour éviter les doublons, les différentes sociétés de discipline doivent se coordonner pour clarifier les questions qui touchent à plusieurs spécialisations. De cette manière, le corps médical consolidera sa position, grâce à laquelle il apparaîtra comme une instance compétente aux yeux de ses partenaires, de l'administration, du monde politique et de l'opinion publique.

Dr Christoph Bosshard, membre du Comité central de la FMH, responsable du domaine Données, démographie et qualité 